\title{
Effect of Nicotine- and Tar-Removed Cigarette Smoke Extract on Cancer Metastasis
}

Yuta Takahashi, Ph.D. ${ }^{1,2,3}$, Takuya Araki, Ph.D. ${ }^{2,4 *}$, Ayumu Nagamine, Ph.D. ${ }^{1,2}$, Hideaki Yashima, Ph.D. ${ }^{4}$, Daisuke Nagano, Ph.D. ${ }^{2}$, Kyoko Obayashi, Ph.D. ${ }^{1}$ and

\section{Koujirou Yamamoto, Ph.D. ${ }^{2,4}$}

${ }^{1}$ Education Center for Clinical Pharmacy, Faculty of Pharmacy, Takasaki University of Health and Welfare, 60 Nakaorui-machi, Takasaki, Gunma, 370-0033, Japan.

${ }^{2}$ Department of Clinical Pharmacology and Therapeutics, Gunma University Graduate School of Medicine, 3-39-22 Showa-machi, Maebashi, Gunma 371-8511, Japan.

${ }^{3}$ Department of Pharmacology, School of Pharmacy and Pharmaceutical Sciences, Mukogawa

Women's University, 11-68 Koshien Kyuban-cho, Nishinomiya, Hyogo 663-8179, Japan.

${ }^{4}$ Department of Pharmacy, Gunma University Hospital, 3-39-15 Showa-machi, Maebashi, Gunma 371-8511, Japan.

Submitted : 3 Oct 2021, Revised : 14 Oct 2021, Accepted : 26 Oct 2021, Published : 3 Nov 2021

\section{Abstract}

Cigarette smoking is known to impact the promotion of carcinogenesis and tumor metastasis. On the other hand, some components in smoke were found to have health-promoting effects, and cancer suppressor effects of components in tobacco smoke have attracted attention. Although some studies showed the cancer suppressive effect of cigarette smoke extract (CSE) in vitro study, the effect of CSE administration on cancer is controversial. In this study, we investigated the effect of CSE-administration on tumor metastasis in a spontaneous tumor metastasis model using B16-BL6 cells, which is more clinical conditions. C57BL/6NCr mice were subcutaneously inoculated B16-BL6 cells into the footpad of the right rear leg. CSE was intraperitoneally administrated for 28 days from the day of inoculation. At 2 weeks after inoculation, the primary focus was excised. Subsequently, survival days of the mice were recorded to determine the effect of CSE-administration on spontaneous metastasis. The effect of CSE, $\alpha, \beta$-unsaturated ketones, and aldehydes on B16-BL6 cell invasiveness were confirmed by matrigel invasion assay. Survival days of mice injected with $100 \%$ CSE was significantly shortened than that of control. B16-BL6 cell invasiveness was accelerated by the treatment with $0.1 \% \mathrm{CSE}$ and $3 \mu \mathrm{M}$ of crotonaldehyde. Intraperitoneal CSE-administration may progress spontaneous metastasis of B16-BL6 cells via enhancement of B16-BL6 cell invasiveness. As the cause, we found that crotonaldehyde contained in CSE may enhance the invasion ability of cancer cells. To clarify the cancer-suppressing effect of tobacco components, the effect of crotonaldehyde-removed CSE on tumor should be assessed in detail.

Keywords: cigarette smoke extract (CSE), metastasis, crotonaldehyde (CA), B16-BL6 mouse melanoma cells, invasion 


\section{Introduction}

Cigarette smoking is widely known to impact the promotion of carcinogenesis and tumor metastasis in several cancer types [14]. Especially in lung cancer, it is reported that smoking rate and carcinogenic risk are significantly correlated, and about $70 \%$ of lung cancer were attributed to smoking in a study of Japanese men with a relatively low increase in cancer risk due to smoking [1]. Similarly, for the esophagus, liver, pancreas, head and neck, cervix, and bladder cancers, smoking has been reported to be one of the significant carcinogenic risks [5-10].

The Mainstream of cigarette smoke is composed of about 4,300 kinds of particulate components and about 1,000 kinds of gas components [11]. Of these, approximately 70 components have been reported to be carcinogenic or may have some adverse health effects [12]. In a study of cigarette smoke, cigarette smoke exposure was reported to increase lung metastasis and tumor volume in colon and pancreatic cancer cell lines in mice [13, 14]. Nicotine, known as one of the causes of tobacco dependence, is also known to impact tumor growth and progression [15, 16], and Nguyen et al. reported that nicotine promotes the proliferation and migration of melanoma cell line by regulating PD-L1 expression via $\alpha 9$ nicotinic acetylcholine receptors [17].

On the other hand, Sayed et al. found health-promoting components in cigarette smoke [18], and reported that some components, such as cembratriene-4, 6-diol and methyl vinyl ketone (MVK), have a tumor-suppressing activity [19-22]. Thus, cancer suppressor effects of components in tobacco smoke have attracted attention, and several studies about cancer suppression using tobacco smoke have been conducted. Saito et al. showed that the tumor-promoting activity of 12-O-tetradecanoylphorbol 13acetate, one of the phorbol esters, was suppressed by cembratriene-4, 6-diol, isolated from tobacco smoke concentrate, in vitro study [20], and Sayed et al. reported that cembratriene-4, 6-diol could inhibit tumor cell invasion [19]. In addition, our group has also reported that the pretreatment of highly metastatic B16-BL6 mouse melanoma (B16BL6) cells with nicotine- and tar-removed cigarette smoke extract (CSE) could reduce the number of lung nodules of B16-BL6 cells in hematogenous lung metastasis model mice injected with B16-BL6 cells through the tail vein [21]. Furthermore, as a study to evaluate the effect of CSE on tumor metastasis in mice, Hatai et al. conducted an in vivo study on the tumor-suppressive activity of intraperitoneal (i.p.) administration of CSE in mice and showed the possibility of i.p. administration of CSE to suppress liver metastasis in a model of transsplenic liver metastasis using colon-26 [22]. They further searched for components involved in cancer metastasis suppression and found MVK, an $\alpha, \beta$ unsaturated ketone contained in tobacco smoke, can suppress metastasis through suppression of invasion of colon-26 cells [22]. However, in the study by Hatai et al., the survival time of mice was not found to be prolonged by CSE administration [22], and the cancer metastasis-suppressing effect of CSE administration on living organisms has 
not been clarified yet. Furthermore, since CSE was administered intraperitoneally in their study, it cannot be ruled out that liver metastasis may have been suppressed by direct exposure of CSE to the primary organ of tumor cell engraftment, spleen. Thus, the cancer metastasis-suppressing effect of CSE administration on living organisms remains controversial.

In this study, we investigated the effect of CSE on tumor metastasis a spontaneous tumor metastasis model in which B16-BL6 cells were seeded subcutaneously in the footpad and subsequently developed lung metastatic nodules, which is more clinical conditions and can rule out the effects of direct exposure of CSE to the primary organ of tumor cell engraftment.

\section{Materials and Methods}

\subsection{Materials}

Frontier Lights brand cigarettes containing $1 \mathrm{mg}$ of tar and $0.1 \mathrm{mg}$ of nicotine per cigarette, were purchased from Japan Tobacco, Inc. (Tokyo, Japan). Cambridge filters, used to remove almost all particles and nicotine from cigarette smoke, were obtained from Heinr. Borgwaldt $\mathrm{GmbH}$ (Hamburg, Germany). Fetal bovine serum (FBS) was from BioWest Co. (Nuaillé, France). EDTA trypsin solution (EDTA: 2.2 $\mathrm{mM}$, trypsin: $0.25 \%$ ) was from Mediatech, Inc. (Manassas, VA, USA). Penicillin/streptomycin solution (penicillin: 50,000 U/mL, streptomycin: $50 \mathrm{mg} / \mathrm{mL}$ ) was from Cosmo Bio Co., Ltd. (Tokyo, Japan). Dulbecco's modified Eagle's medium (DMEM) with L-glutamine was from Invitrogen Corp. (Carlsbad, CA, USA).
Dulbecco's phosphate-buffered saline without calcium and magnesium [DPBS (-)] was from Nissui Pharmaceutical Co., Ltd. (Tokyo, Japan). Growth factor-reduced Matrigel matrix and FALCON cell culture inserts were from Becton Dickinson Labware (Bedford, MA, USA).

\subsection{Preparation of CSE}

The CSE was prepared according to the method described in a previous report [21]. Briefly, CSE was obtained by bubbling the filtered mainstream of smoke (gas phase) into DPBS (-) (1 mL per 3 cigarettes). As a filter, Cambridge filter was used to remove the particle phase containing tar and nicotine. The suction speed was kept constant (1 $\mathrm{L} / \mathrm{min}$ ) using a suction pump (Nippon Rikagaku Kikai Co., Ltd., Tokyo, Japan), and smoke was bubbled for $1 \mathrm{~min}$. The CSE contained DPBS (-) solution was immediately filtrated with a $0.22 \mu \mathrm{m}$ filter. The filtered solution, $100 \% \mathrm{CSE}$, was stored at $-80^{\circ} \mathrm{C}$ until use and diluted to various concentrations with DPBS (-) at the time of use.

\subsection{Animals}

Specific pathogen-free male C57BL/6NCr mice (7 weeks old) purchased from Japan SLC, Inc. (Hamamatsu, Japan) were used as metastatic melanoma syngeneic animals. Mice were maintained in an airconditioned room $\left(23 \pm 2^{\circ} \mathrm{C}\right.$ and $60 \pm 10 \%$ humidity) under an artificial 12-hour light/dark cycle (7:00 a.m.- 7:00 p.m.). Food and water were given ad libitum during the experimental period. This study was approved by the Animal Experiment 
Committee of Mukogawa Women's University (Approval No. P-11-2012-06-A), and all procedures followed the Guidelines for the Care and Use of Laboratory Animals at the University.

\subsection{Cells}

A highly metastatic B16-BL6 mouse melanoma cell line was kindly provided by Dr. Futoshi Okada of Tottori University (Yonago, Japan). Cells less than 50 passages were used in all experiments. B16-BL6 cells were cultured in DMEM containing 10\% FBS and $0.1 \%$ penicillin/streptomycin solution in a humidified incubator at $37^{\circ} \mathrm{C}$ in the presence of $5 \% \mathrm{CO}_{2}$.

\subsection{Evaluation of spontaneous metastasis of tumor cells}

Sub-confluent B16-BL6 cells were harvested with EDTA trypsin solution and resuspended in DPBS $(+)$ to the appropriate concentrations. Fifty microliters of cell suspension $\left(2 \times 10^{7}\right.$ cells $\left./ \mathrm{mL}\right)$ were subcutaneously injected into the footpad of the right hind leg of syngeneic C57BL/6NCr mice. Two weeks after the inoculation, the mice were anesthetized with diethyl ether and the enlarged primary tumor was excised. After 4 weeks from tumor inoculation, 10, 30, and 100\% CSE were administrated with i.p. to the mice at the dose of $16 \mathrm{~mL} / \mathrm{kg}$ daily. As a control, DPBS (-) was administered. The survival of the mice in each group was then followed up for 100 days and the date of death was recorded. The survival duration was determined as the number of days after tumor cell inoculation. Dead mice were dissected for confirmation of tumor metastases. Each group contained 7 animals at the start of the experiment.

\subsection{Proliferation assay}

B16-BL6 cells were seeded on a 12 well plate at $1 \times 10^{5}$ cells/well. Then, cells were treated with CSE $(0.01,0.03$ and $0.1 \%$ as final concentration), $\operatorname{MVK}(1,3,10 \mu \mathrm{M})$, $\mathrm{CA}(1,3,10 \mu \mathrm{M}), \operatorname{ACR}(1,3,10 \mu \mathrm{M})$ or DPBS(-). After $72 \mathrm{~h}$ of exposure to CES, cells were collected and the number of cells in each well was determined using a coulter counter.

\subsection{Matrigel invasion assay}

B16-BL6 cells were resuspended in FBS-free DMEM to obtain a concentration of $4.0 \times 10^{5}$ cells $/ \mathrm{mL}$, and $500 \mu \mathrm{L}$ of cell suspension was added to the upper layer within the cell culture insert coated with Matrigel on the filter. To the lower layer, DMEM containing fibronectin as a chemoattractant was added. After $24 \mathrm{~h}$ of incubation with CSE $(0.01,0.03$ and $0.1 \%$ as final concentration), $3 \mu \mathrm{M}$ of $\mathrm{MVK}, 3 \mu \mathrm{M}$ of CA, $3 \mu \mathrm{M}$ of ACR or $0.1 \%$ of DPBS(-). Uninfiltrated cells, which remain on the top of the filter, were wiped with a swab. The infiltrated cells on the bottom surface of the filter were Giemsa stained and counted under a microscope.

\subsection{Statistical Analyses}

Data are expressed as the mean \pm S.E. Survival data were analyzed by the log-rank test. Data from in vitro experiments were analyzed by Dunnett's test. Statistical analyses were performed using the Graphpad Prism 4 software package (Graphpad 
Software, Inc., San Diego, CA, USA). A difference was considered significant when $p<0.05$.

\section{Results}

\subsection{Effect of intraperitoneal CSE} administration on survival time of spontaneous metastasis model mice

Although administration of CSE with a concentration of under $30 \%$ did not affect the survival time of mice, the survival time was significantly shortened by administration of 100\% CSE compared to controls (Fig. 1). There was no significant change in the weight and thickness of the primary tumor collected 14 days after inoculation of B16BL6 cells in each group (data not shown).

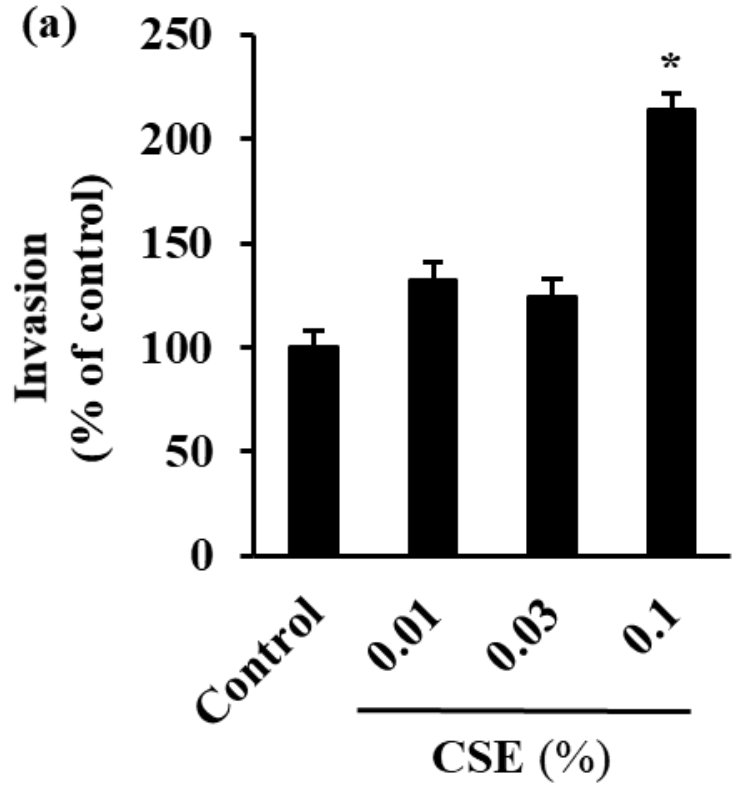

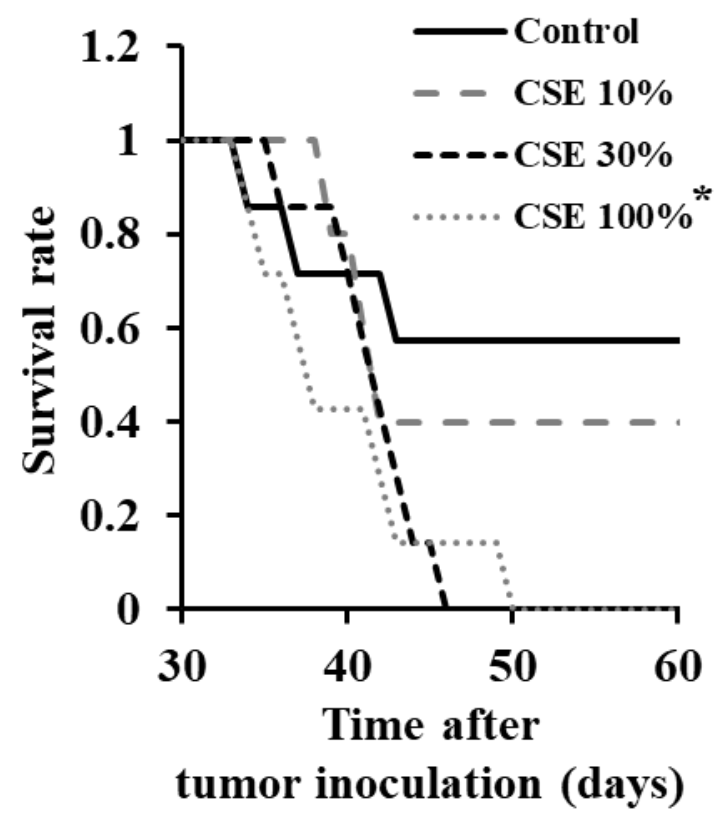

Figure 1. Effect of intraperitoneal CSE administration on survival time of spontaneous metastasis model mice $* P<0.05$ vs control $(\mathrm{n}=5-7)$.

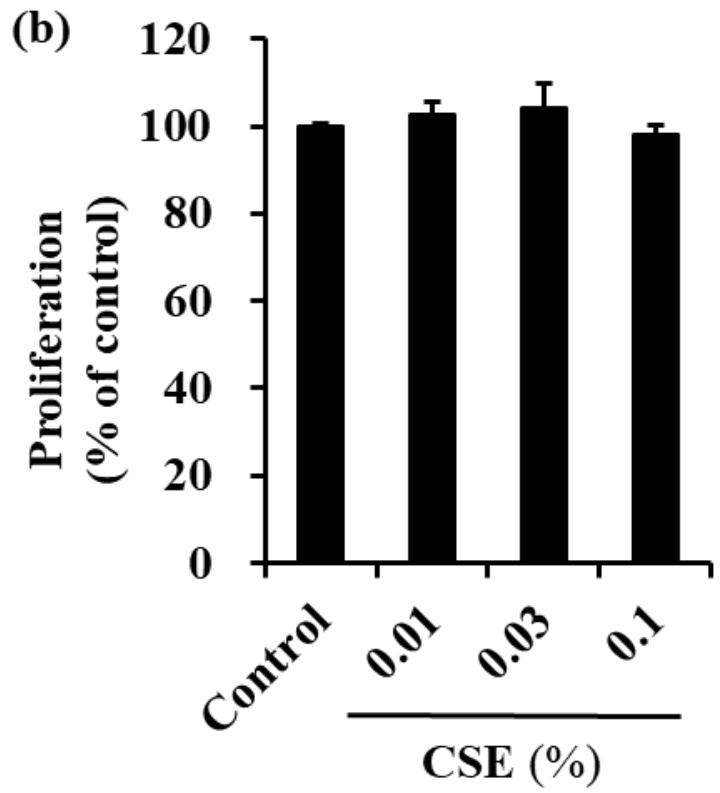

Figure 2. Effect of CSE exposure on the (a) invasion and (b) proliferation of B16-BL6 cells. $* P<0.05$ vs control ((a) $\mathrm{n}=3$ and (b) $\mathrm{n}=6$, respectively). 
3.2. Effect of CSE on invasion and proliferation of B16-BL6 cells

Invasiveness was significantly increased in the $0.1 \%$ CSE exposure group compared to the control (Fig. 2a). On the other hand, the cell proliferation rate was not affected by CSE exposure (Fig. 2b).

\subsection{Effect of MVK, ACR, and CA on B16-}

\section{BL6 cell invasion}

CA increased invasiveness of B16-BL6 cells about 4-fold compared to the control, though ACR and MVK did not show a significant effect on the invasiveness of B16BL6 cells (Fig. 3).

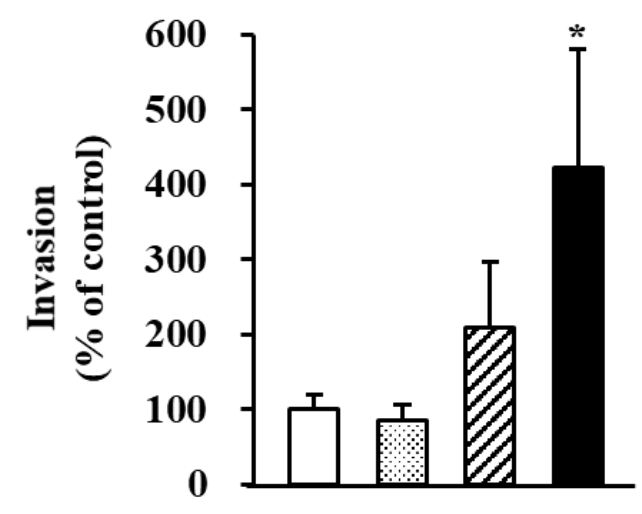

aControl $\quad \mathrm{MVK} 3 \mu \mathrm{M}$

$\square \mathrm{ACR} 3 \mu \mathrm{M} \square \mathrm{CA} 3 \mu \mathrm{M}$

Figure 3. Effect of MVK, ACR, and CA on the invasion of B16-BL6 cells. $* P<0.05$ vs control $(n=5-6)$.

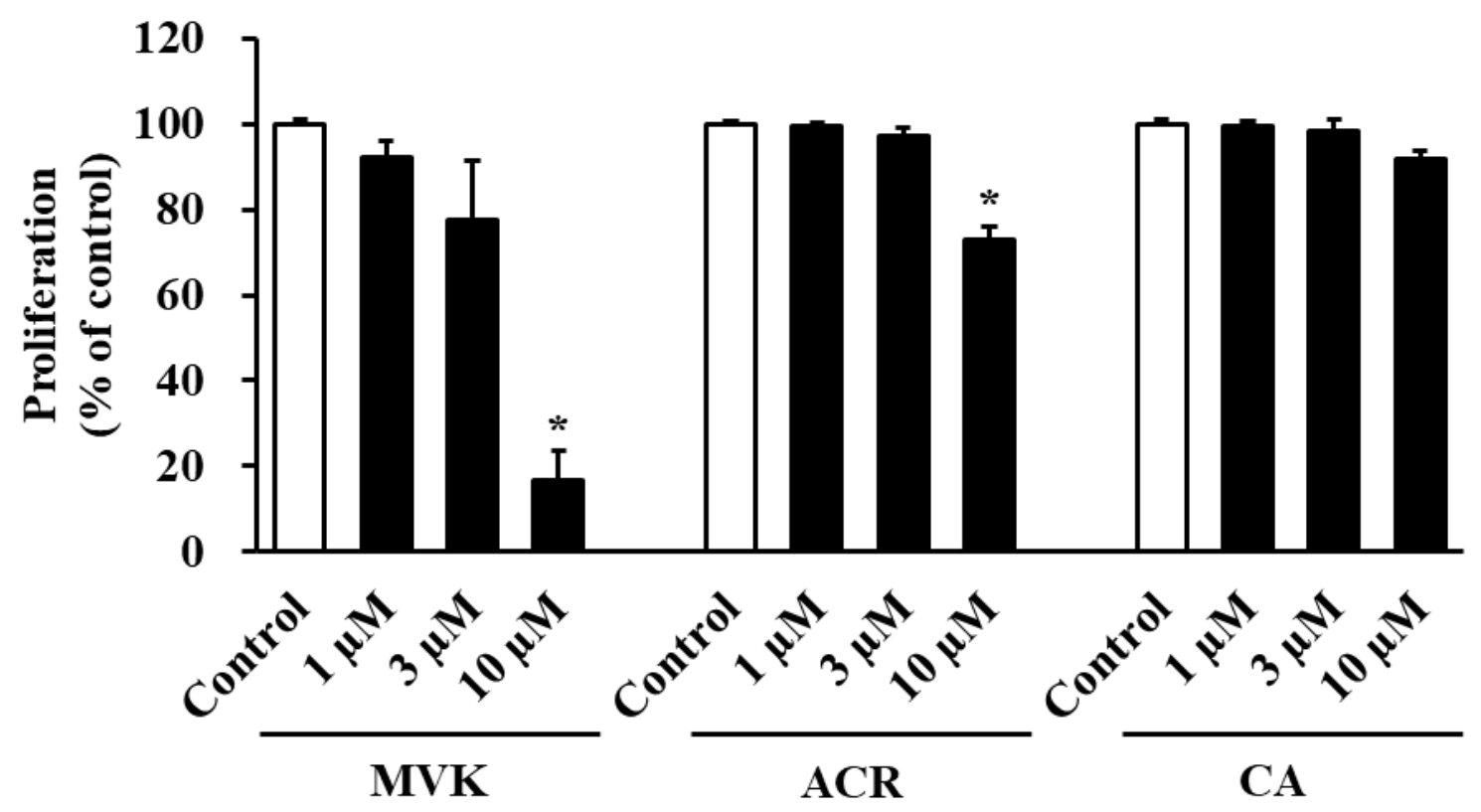

Figure 4. Effect of MVK, ACR, and CA on the proliferation of B16-BL6 cells. ${ }^{*} \mathrm{P}<0.05$ vs control $(n=4)$.

\subsection{Effect of MVK, ACR, and CA on B16-} BL6 cell proliferation

The exposure of $10 \mu \mathrm{M}$ of MVK or ACR significantly suppressed the proliferation of B16-BL6 cells, whereas 3 $\mu \mathrm{M}$ or under of MVK and ACR did not affect the proliferation rate of B16-BL6 cells, and
CA did not inhibit proliferation regardless of exposure concentration (Fig. 4).

\section{Discussions}

In this study, we assessed the effect of intraperitoneal administration of CSE on tumor metastasis using a spontaneous cancer 
metastasis model in which B16-BL6 cells are disseminated subcutaneously in the footpad as primary cancer. As a result, as Hatai et al. showed in a study on intraperitoneal administration of CSE to transsplenic liver metastasis model mice using colon-26 cells, CSE administration could not prolong the survival time of mice [22], but rather significantly shortened the survival of C57BL/6NCr mice when 100\% CSE was used. Since Hatai et al. have reported that intraperitoneal administration of $100 \%$ CSE was not toxic to non-cancer-planted mice [22], the shortening of survival time by $100 \%$ CSE administration confirmed in this study was considered to be the result of CSE affecting the proliferation and metastasis of B16-BL6 cells. In addition, because the primary lesion was resected 14 days after cancer cell dissemination and the thickness or weight of the primary lesion did not change at that point, the shortened survival time was considered to be caused by the effect of CSE on cancer metastasis rather than the primary lesion. So, we assessed the effect of CSE and its major components on the invasion and proliferation of cancer cells to find the cause of the shortened survival time. First, we analyzed the effect of CSE on cancer cell proliferation and infiltration. As a result, although CSE exposure did not affect cancer cell proliferation as Hatai reported, CSE promoted cancer cell invasion, which was different from the report by Hatai et al. These results suggested that the decrease in survival time by CSE may be due to an increase in invasion ability of B16-BL6 cells.

Next, to clarify the causative agent of this invasiveness promotion, we assessed the effects of MVK, ACR, and CA, which are $\alpha$, $\beta$-unsaturated ketones and aldehydes and considered as main components of CSE, on the invasion of B16-BL6 cells. As a result, while those substances did not affect the proliferation of cancer cells, the invasion ability of B16-BL6 cells was significantly increased by CA. From these results, it was considered that $\mathrm{CA}$ was involved in the decrease in survival time of CSEadministered mice through the promotion of B16-BL6 cell invasion. Although CA has been reported to have carcinogenicity and lung cell injury [23, 24], its impact on the invasion ability of tumors has not been reported. To the best of our knowledge, this is the first report that CA has significantly improved the infiltration capacity of cancer cells.

Despite preparing CSE by a method shown in Hatai's report, CSE enhanced cancer cell invasion in our study, while CSE showed inhibitory efficacy on cancer invasion in Hatai's report [22]. As the reason for this difference, it was considered to be a large difference in CA concentration in CSE in addition to the difference in cell lines. Slight changes in conditions when extracting CSE or a difference in the lot of tobacco to use may cause a difference in the components contained in tobacco smoke. We need to examine in detail the factor affecting $\mathrm{CA}$ content in smoke and differences in CA content in smoke between lots of tobacco. In any case, in order to assess the tumorsuppressive efficacy of CSE in detail, it was considered important to remove CA, which 
has an enhancing effect on cancer invasion ability, not only nicotine and tar.

Although CSE suppressed metastasis of cancer cells in the previous study, CSE could not suppress metastasis in our study, and the cause of the discrepancy in these results is thought to be due to the difference in the level of CSE exposure to cancer cells. In vitro study about the effect of tobacco smoke, CSE was exposed directly to cancer cells, and cancer cells are exposed to CSE with high level. Similarly, in the study of Hatai et al., intraperitoneal administration of CSE could expose the spleen, where cancer cells first engrafted in the model, and cancer cells to high levels of CSE. And this high level of CSE exposure can have a high metastasis-suppressing effect. Bourgeois et al. reported that cellular response to CSE exposure is dependent not only on the nominal concentration of CSE, but also on specific experimental variables, including the total cell number, and the volume of CSE solution used [25]. They also reported that the effective dose of CSE is more accurately related to the amount of bioavailable chemicals per cell. Similarly, Lee et al. reported that CSE-induced cytotoxicity was reduced at high cell densities [26]. Based on these reports, it is considered that the metastasis of cancer cells was not suppressed in this study because the transfer of CSE to the subcutaneous footpad where cancer cells engrafted was insufficient. In this study, we planned to weigh the lung, which is the site of metastasis, to evaluate the effect of CSE on metastasis. However, because of severe damage to the lung due to cancer metastasis, lung tissue could not be collected, and the cancer metastasis suppressing effect of CSE could not be evaluated in detail. In addition, since CA could not be removed from CSE, the effect of CA-removed CSE on tumor could not be assessed. We need to investigate in detail whether exposure to CA-removed CSE can suppress cancer.

\section{Conclusion}

In conclusion, intraperitoneal administration of CSE significantly shortened the survival time of spontaneous lung metastasis model $\mathrm{C} 57 \mathrm{BL} / 6 \mathrm{NCr}$ mice seeded with B16-BL6 cells. As the cause, we found that CA contained in CSE may enhance the invasion ability of cancer cells. To the best of our knowledge, this is the first report that CA has significantly improved the infiltration capacity of cancer cells. In the future, to clarify the cancer-suppressing effect of tobacco components, it was considered important to evaluate the cancer suppression effect of CA-removed CSE in detail and to establish a delivery system, which can transfer the components in CSE to cancer tissues efficiently.

\section{Acknowledgement}

We thank Prof. Kazuki Nakamura, Assoc. Prof. Noriko Yoshikawa, and Lecturer Shizuyo Horiyama of Mukogawa Women's University for their advice on research.

\section{References}

1. Sobue $T$, Yamamoto $\mathrm{S}$, Hara $\mathrm{M}$, Sasazuki S, Sasaki S, Tsugane S, et al. Cigarette smoking and subsequent risk of lung cancer by histologic type in 
middle-aged Japanese men and women: the JPHC study. Int J Cancer. 2002;99(2):245-51.

2. Moreira DM, Aronson WJ, Terris MK, Kane CJ, Amling CL, Cooperberg MR, et al. Cigarette smoking is associated with an increased risk of biochemical disease recurrence, metastasis, castration-resistant prostate cancer, and mortality after radical prostatectomy: results from the SEARCH database. Cancer 2014;120(2):197-204.

3. Yahagi M, Tsuruta M, Hasegawa $\mathrm{H}$, Okabayashi K, Toyoda N, Iwama N, et al. Smoking is a risk factor for pulmonary metastasis in colorectal cancer. Colorectal Dis. 2017;19(9):O322-8.

4. Li H, Terry MB, Antoniou AC, Phillips KA, Kast K, Mooij TM, et al. Alcohol Consumption, Cigarette Smoking, and Risk of Breast Cancer for BRCA1 and BRCA2 Mutation Carriers: Results from The BRCA1 and BRCA2 Cohort Consortium. Cancer Epidemiol Biomarkers Prev. 2020;29(2):368-378.

5. Liu L, Huang C, Liao W, Chen S, Cai S. Smoking behavior and smoking index as prognostic indicators for patients with esophageal squamous cell carcinoma who underwent surgery: A large cohort study in Guangzhou, China. Tob Induc Dis. 2020;18:9.

6. Pang Q, Qu K, Zhang J, Xu X, Liu S, Song $\mathrm{S}$, et al. Cigarette smoking increases the risk of mortality from liver cancer: A clinical-based cohort and meta-analysis. J Gastroenterol
Hepatol. 2015;30(10):1450-60.

7. Matsuo K, Ito H, Wakai K, Nagata C, Mizoue T, Tanaka K, et al. Cigarette smoking and pancreas cancer risk: an evaluation based on a systematic review of epidemiologic evidence in the Japanese population. Jpn J Clin Oncol. 2011;41(11):1292-302.

8. Koyanagi YN, Matsuo K, Ito H, Wakai K, Nagata C, Nakayama $T$, et al. Cigarette smoking and the risk of head and neck cancer in the Japanese population: a systematic review and meta-analysis. Jpn J Clin Oncol. 2016;46(6):580-95.

9. Sugawara Y, Tsuji I, Mizoue T, Inoue M, Sawada N, Matsuo K, et al. Cigarette smoking and cervical cancer risk: an evaluation based on a systematic review and meta-analysis among Japanese women. Jpn J Clin Oncol. 2019;49(1):77-86.

10. Masaoka H, Matsuo K, Ito H, Wakai K, Nagata C, Nakayama T, et al. Cigarette smoking and bladder cancer risk: an evaluation based on a systematic review of epidemiologic evidence in the Japanese population. Jpn J Clin Oncol. 2016;46(3):273-83.

11. Rodgman A, Perfetti TA. The Chemical Components of Tobacco and Tobacco Smoke Second Edition. Boca Raton: CRC Press, 2013.

12. IARC Working Group on the Evaluation of Carcinogenic Risks to Humans. A review of human carcinogens: personal habits and indoor combustions. IARC Monogr 
Eval Carcinog Risks Hum 2012;100E:1-579.

13. Makino A, Tsuruta M, Okabayashi K, Ishida T, Shigeta K, Seishima R, et al. The Impact of Smoking on Pulmonary Metastasis in Colorectal Cancer. Onco Targets Ther. 2020;13:9623-9.

14. Momi N, Ponnusamy MP, Kaur S, Rachagani S, Kunigal SS, Chellappan $\mathrm{S}$, et al. Nicotine/cigarette smoke promotes metastasis of pancreatic cancer through $\alpha 7 \mathrm{nAChR}-$ mediated MUC4 upregulation. Oncogene. 2013;32(11):1384-95.

15. Wu SY, Xing F, Sharma S, Wu K, Tyagi A, Liu Y, et al. Nicotine promotes brain metastasis by polarizing microglia and suppressing innate immune function. $\mathrm{J}$ Exp Med. 2020;217(8):e20191131.

16. Tyagi A, Sharma S, Wu K, Wu SY, Xing F, Liu Y, et al. Nicotine promotes breast cancer metastasis by stimulating N2 neutrophils and generating premetastatic niche in lung. Nat Commun. 2021;12(1):474.

17. Nguyen HD, Liao YC, Ho YS, Chen LC, Chang HW, Cheng TC, et al. The $\alpha 9$ Nicotinic Acetylcholine Receptor Mediates Nicotine-Induced PD-L1 Expression and Regulates Melanoma Cell Proliferation and Migration. Cancers (Basel). 2019;11(12):1991.

18. El Sayed KA, Sylvester PW. Biocatalytic and semisynthetic studies of the anticancer tobacco cembranoids. Expert Opin Investig Drugs. 2007;16(6):877-87.

19. El Sayed KA, Laphookhieo S, Baraka
HN, Yousaf M, Hebert A, Bagaley D, et al. Biocatalytic and semisynthetic optimization of the anti-invasive tobacco (1S,2E,4R,6R,7E,11E)-2,7,11cembratriene-4,6-diol. Bioorg Med Chem. 2008;16(6):2886-93.

20. Saito Y, Takizawa H, Konishi S, Yoshida D, Mizusaki S. Identification of cembratriene-4,6-diol as antitumorpromoting agent from cigarette smoke condensate. Carcinogenesis. 1985;6(8):1189-94.

21. Takahashi Y, Horiyama S, Kimoto Y, Yoshikawa N, Kunitomo M, Kagota S, et al. Inhibitory effect of cigarette smoke extract on experimental lung metastasis of mouse melanoma by suppressing tumor invasion. Pharmacology and Pharmacy. 2012;3(3):324-9.

22. Hatai M, Yoshikawa N, Kinoshita E, Horiyama S, Kagota S, Shinozuka K, et al. Invasion-inhibiting Effects of Gaseous Components in Cigarette Smoke on Mouse Rectal Carcinoma Colon-26 Cells. In Vivo. 2018;32(3):493-7.

23. IARC Monographs Vol 128 group. Carcinogenicity of acrolein, crotonaldehyde, and arecoline. Lancet Oncol. 2021;22(1):19-20.

24. Li S, Wei P, Zhang B, Chen K, Shi G, Zhang Z, et al. Apoptosis of lung cells regulated by mitochondrial signal pathway in crotonaldehyde-induced lung injury. Environ Toxicol. 2020;35(11):1260-73.

25. Bourgeois JS, Jacob J, Garewal A, 
Ndahayo R, Paxson J. The Bioavailability of Soluble Cigarette Smoke Extract Is Reduced through Interactions with Cells and Affects the Cellular Response to CSE Exposure. PLoS One. 2016;11(9):e0163182.

26. Lee YC, Chuang CY, Lee PK, Lee JS,
Harper RW, Buckpitt AB, et al. TRXASK1-JNK signaling regulation of cell density-dependent cytotoxicity in cigarette smoke-exposed human bronchial epithelial cells. Am J Physiol Lung Cell Mol Physiol. 2008;294(5):L921-31. 